OPEN ACCESS

Edited by:

Virginie Lafont,

Institut National de la Santé et de la

Recherche Médicale (INSERM),

France

Reviewed by:

Monika C. Brunner-Weinzierl,

University Hospital Magdeburg,

Germany

Thorbald Van Hall,

Leiden University, Netherlands

*Correspondence:

Raul M. Torres

raul.torres@cuanschutz.edu

Specialty section:

This article was submitted to

Cancer Immunity

and Immunotherapy,

a section of the journal

Frontiers in Immunology

Received: 01 February 2020 Accepted: 30 November 2020

Published: 27 January 2021

Citation:

Mathew D and Torres RM (2021)

Lysophosphatidic Acid Is an Inflammatory Lipid Exploited by Cancers for Immune Evasion via Mechanisms Similar and Distinct

From CTLA-4 and PD-1.

Front. Immunol. 11:531910. doi: 10.3389/fimmu.2020.531910

\section{Lysophosphatidic Acid Is an Inflammatory Lipid Exploited by Cancers for Immune Evasion via Mechanisms Similar and Distinct From CTLA-4 and PD-1}

\author{
Divij Mathew and Raul M. Torres*
}

Department of Immunology \& Microbiology, University of Colorado School of Medicine, Aurora, CO, United States

Immunological tolerance has evolved to curtail immune responses against self-antigens and prevent autoimmunity. One mechanism that contributes to immunological tolerance is the expression of inhibitory receptors by lymphocytes that signal to dampen immune responses during the course of an infection and to prevent immune-mediated collateral damage to the host. The understanding that tumors exploit these physiological mechanisms to avoid elimination has led to remarkable, but limited, success in the treatment of cancer through the use of biologics that interfere with the ability of cancers to suppress immune function. This therapy, based on the understanding of how T lymphocytes are normally activated and suppressed, has led to the development of therapeutic blocking antibodies, referred to as immune checkpoint blockade, which either directly or indirectly promote the activation of CD8 T cells to eradicate cancer. Here, we highlight the distinct signaling mechanisms, timing and location of inhibition used by the CTLA-4 and PD-1 inhibitory receptors compared to a novel inhibitory signaling axis comprised of the bioactive lipid, lysophosphatidic acid (LPA), signaling via the LPA5 receptor expressed by CD8 T cells. Importantly, abundant evidence indicates that an LPA-LPA5 signaling axis is also exploited by diverse cancers to suppress $T$ cell activation and function. Clearly, a thorough molecular and biochemical understanding of how diverse $T$ cell inhibitory receptors signal to suppress $T$ cell antigen receptor signaling and function will be important to inform the choice of which complimentary checkpoint blockade modalities might be used for a given cancer.

Keywords: CD8 T cell, cancer, lysophophatidic acid, LPAR5, inhibitory receptor and ligand

\section{INTRODUCTION}

Lipid biology in the context of tumor immunity remains vastly unexplored. However its role in modulating inflammation has been used for centuries (1), which has led to pharmaceutical development of nonsteroidal anti-inflammatory drugs (NSAIDs), like COX-2 inhibitors, that inhibit the generation of prostaglandin and thromboxane lipids. Interestingly, COX-2 inhibitors also lower cancer promoting inflammation and drive type I immunity, demonstrating a unique role of lipids 
affecting the anti-tumor response (2). This review seeks to highlight another bioactive lipid, lysophosphatidic acid (LPA), and its role in dampening tumor immunity. Unlike COX-2, LPA suppresses the anti-tumor response in a cell intrinsic manner by signaling via an LPA receptor (LPAR) similar to checkpoint receptors. Furthermore, as LPA is found systemically, and in all tissues, we speculate mechanisms of suppression mediated by LPARs are similar to CTLA and PD-1 in inhibiting T cell antigen receptor (TCR) signaling, yet via distinct signaling pathways. Specifically, LPA signaling has been shown to suppress $\mathrm{T}$ cell TCR $\mathrm{Ca}^{2+}$ signaling which inhibits naïve $\mathrm{T}$ cell activation in secondary lymphoid organs. Additionally, as tumors can produce LPA at higher concentrations than adjacent tissue, this tumor-derived LPA also inhibits $\mathrm{T}$ cell effector function therefore representing a checkpoint in $\mathrm{T}$ cell function similar to that mediated by CTLA4 and PD-1. Therefore, as a lipid, LPA/LPAR modulation of immune responses has functional similarities to other checkpoint molecules like PD-1/PD-L1 or CTLA-4/CD80 yet remains unique to other soluble or cell-associated protein-protein interactions.

\section{CTLA-4: SUPPRESSION OF EARLY T CELL ACTIVATION}

CTLA-4 is a major inhibitory receptor expressed by CD8 T cells and was an initial therapeutic target given that CTLA-4 is expressed following TCR engagement by naïve T cells and continues to rise until maximum expression at $48 \mathrm{~h}$ (3). Compared to the CD28 T cell costimulatory receptor, CTLA-4 displays significantly higher affinity for the CD80 and CD86 coreceptors expressed by antigen presenting cells (APCs). Thus, when expressed, CTLA-4 effectively sequesters CD80 and CD86 away from CD28 and prevents co-stimulatory signaling activity for the T cell antigen receptor (TCR) (4). Furthermore, CTLA-4 interaction with CD80/CD86 can also lead to transendocytosis, the physical capture of these ligands and removal from the surface of APCs, thereby limiting total levels of available ligands for CD28 and subsequent co-stimulation (4). The physical sequestration of CD28 ligands ultimately prevents optimal TCR signaling by CD8 T cells and is an important mechanism by which CTLA-4 suppresses $\mathrm{T}$ cell function (5). This process of CTLA-4-mediated CD80/CD86 transendocytosis is seen in all $\mathrm{T}$ cell subsets but particularly in regulatory $\mathrm{T}$ cells contributing to the suppressive abilities of this $\mathrm{T}$ cell subpopulation (5). Notably, deletion of the CTLA-4 cytoplasmic tail has been reported not to change its ability to suppress $\mathrm{T}$ cell proliferation indicating CTLA-4 intracellular signaling is not necessary for all $\mathrm{T}$ cell inhibition (6). Furthermore, agonist signaling via CTLA-4 does not induce significant changes in $\mathrm{T}$ cell gene expression also suggesting a mechanism of inhibition that does not rely on intracellular signaling (7). However, coimmunoprecipitation of CTLA-4 in a T cell hybridoma reveals interaction with PKC- $\eta$ (8). Tregs depleted of PKC- $\eta$ have compromised suppressive function both in vitro and in vivo thus suggesting a cell-intrinsic signaling role for CTLA-4 (8). The significance of immune inhibition provided by CTLA-4 is highlighted by the fact that $\mathrm{Ctla}^{-/-}$mice survive only for the first 3-4 weeks and then die as a result of massive lymphocytic infiltration and destruction of major organs (9-11). Thus, a CTLA-4-deficiency leads to uncontrolled expansion of CD4 $\mathrm{T}$ cells, including autoreactive $\mathrm{T}$ cells that subsequently damage host organs thus indicating that CTLA-4 is required for appropriate maintenance of peripheral tolerance.

Under normal conditions, $\mathrm{T}$ cells that develop in the thymus and recognize self-antigens with relatively high affinity are culled from development by mechanisms of central tolerance. Given that all tumors originate from normal cells, the (non-mutated) tumor antigens they express are essentially self-antigens and central tolerance similarly deletes host CD8 T cells able to recognize tumor antigens with high affinity. Nevertheless, central tolerance is not absolute and does not remove all selfreactive $\mathrm{T}$ cells, especially those that display weak affinity for selfantigens. These weakly autoreactive $\mathrm{T}$ lymphocytes emigrate to the periphery and are further restrained by mechanisms of peripheral tolerance. Indeed, early experiments designed to block the CTLA-4 inhibitory receptor to improve the endogenous self/tumor host response against a murine 51BLim10 colon cancer resulted in immediate rejection of the tumor (12). Accordingly, anti-CTLA-4 blockade therapy has shown success in the clinic, however, this approach also presents with treatment related toxicities such as nausea and fatigue seen in $70-80 \%$ of patients or dermatitis and enterocolitis seen in 5-25\% of patients (13). Recently, anti-CTLA-4 antibodies have been engineered to behave differently during the endosomal trafficking of CTLA-4 to minimize these adverse events. Specifically, ipilimumab harboring tyrosine-to-histidine mutations display increased $\mathrm{pH}$ sensitivity and upon entering the endosomes bound to CTLA-4, disengages from CTLA- 4 in the lysosomes allowing CTLA-4 to recycle to the surface in a LRBA dependent manner which still induces tumor regression with minimal associated adverse events (14). Of note, recent data has suggested that an alternate mechanism by which anti-CTLA4 antibodies act is via the depletion of regulatory T cells $(15,16)$, thus questioning its mechanism of action as a checkpoint blockade therapy and suggesting it may instead act as an antibody depleting therapy. In contrast, while treatment of patients with either ipilimumab or tremelimumab anti-CTLA-4 monoclonal antibodies has led to increased (CD4 and CD8) $\mathrm{T}$ cell tumor infiltration, the number of tumor-infiltrating FOXP3+ regulatory $\mathrm{T}$ cells was not significantly altered (17). Thus, blocking CTLA-4 represents a form of checkpoint blockade that allows for greater primary expansion of effector $\mathrm{T}$ cells; however, the precise mechanism by which CTLA-4 modulates CD8 $\mathrm{T}$ cells in the tumor microenvironment is less certain.

\section{PD-1: CO-OPTED BY THE TUMOR MICROENVIRONMENT}

As CTLA-4 expression is initiated soon after initial $\mathrm{T}$ cell antigen-recognition by naïve $\mathrm{T}$ cells, its mechanism of $\mathrm{T}$ cell 
suppression is considered to be primarily restricted to secondary lymphoid organs where $\mathrm{T}$ cells typically first encounter foreign antigens. However, additional inhibitory receptors are also expressed by CD8 $\mathrm{T}$ cells and able to suppress cytotoxic function at various stages. PD-1 is another inhibitory receptor expressed by activated CD8 T cells and able to dampen effector function and has received abundant attention as a target of immune checkpoint blockade. Unlike CTLA-4, PD-1 harbors both ITIM and ITSM tyrosine-based motif sequences in the cytoplasmic domain (18) that facilitate inhibitory function and arguing for a mechanism of suppression dependent on receptor signaling. While it is generally considered that inhibition mediated by $\mathrm{PD}-1$ is promoted through the recruitment of cytosolic phosphatases, it remains unclear precisely which stimulatory signals are the targets of inhibition. Immunoprecipitation of $\mathrm{CD} 3 \zeta$ shows a $\sim 70 \%$ reduction of phosphotyrosine when TCR and PD-1 are co-ligated in comparison to TCR ligation alone (19). Furthermore, both SHP-1 and SHP-2 are thought to mediate this suppression as both were found to immunoprecipitate with the ITSM domain of PD-1 (17). However, as PD-1 preferentially clusters with CD28 rather than the TCR, this argues that CD28 is the preferential target of PD-1 signaling (20). In fact, using a cellfree FRET-based assay, it was determined that PD-1 selectively recruited SHP-2 which in turn dephosphorylated CD28.

Despite discrepancies in the described mechanism(s) of PD-1 inhibitory action, PD-1 blockade has enjoyed major success in the clinic. This is because a major ligand for PD-1 is PD-L1, whose expression is upregulated by diverse tumors in response to IFN $\gamma$. In fact when examined, $298 \%$ of PD-L1 expressing melanocytes were co-localized with $\mathrm{T}$ cells as opposed to minimal co-localization of T cells with PD-L1-negative tumor cells, suggesting tumor cells express PD-L1 in response to infiltrating $\mathrm{T}$ cells (21). Thus, attenuating this inhibitory signal prevents $\mathrm{T}$ cell suppression and leads to increased cytotoxicity from tumor-specific CD8 T cells. Indeed, the success of anti-PD1/PD-L1 signaling is highlighted by having garnered FDA approval for the treatment of kidney cancers, melanomas, prostate cancers, lung cancers, B cells lymphomas, Hodgkin's lymphoma, urothelial carcinomas, gastric cancers, liver cancers, cervical cancers, and head and neck cancers in the last 5 years (22). Although the majority of evidence suggests the PD-1/PDL1 signaling axis acts primarily at sites of chronic inflammation, recent data provides evidence for a role for $\mathrm{PD}-1$ signaling during the early phases of T cell activation (23). Specifically, upon TCRmediated activation, $\mathrm{PD}-1$ expression by CD8 $\mathrm{T}$ cells is upregulated within $4 \mathrm{~h}$, matching the kinetics of the CD25 activation antigen and preceding cell division, thus arguing for a physiological role for PD-1 during primary activation (23). PDL1 blockade on day 0 and day 3 after LCMV Armstrong infection led to increased granzyme B and mTOR signaling two days later by CD8 T cells (23). Thus, PD-1 signaling appears to suppress T cell function at various stages representing another 'checkpoint' that tumors exploit to escape elimination.

Given the clinical success and limitations of these therapeutic interventions for cancer, additional effort is needed to better understand precisely how $\mathrm{T}$ cell function is regulated by both stimulatory and regulatory signals (24). Ongoing and new research has identified novel protein inhibitory receptors and below we further describe a lipid that signals via a cognate Gprotein coupled receptor (GPCR) to deliver suppressive signals to CD8 $\mathrm{T}$ cells and which ultimately negatively-regulate $\mathrm{T}$ cell function.

\section{LYSOPHOSPHATIDIC ACID}

Lysophosphatidic acid (LPA) is a lysophospholipid structurally similar to sphingosine-1-phosphate (S1P), a lipid that has been well characterized to signal to immune cells and to orchestrate cell trafficking (25). Both lipids share a phosphate head group attached to a glycerol backbone; however, LPA differs by having a single ester linked aliphatic chain whereas S1P has a single amine linked aliphatic chain. On initial discovery both LPA and S1P were considered to be intracellular lipid metabolites and only later were characterized to function as extracellular bioactive lipids that signal to cells expressing cognate G-protein coupled receptors (GPCRs). Extracellular LPA is generated predominantly via the enzymatic activity of Autotaxin (ATX), a secreted ectoenzyme with lysophospholipase D activity that hydrolyzes the abundantly available lysophosphatidylcholine to produce LPA. Although five isoforms of ATX exist through alternative splicing of exons 12, 19, and 21, ATX $\beta$ is the form most expressed in tissue (26). Autotaxin is encoded by ENPP2 and is highly expressed in nervous system as well as considerable expression by stromal and endothelial cells with reduced general expression in most other tissues. Structural studies have indicated that Autotaxin harbors an exposed integrin binding motif and, as a secreted enzyme, Autotoxin is thought to associate with surface-bound integrins $(27,28)$. Thus, current models posit that in certain microenvironments integrin-bound Autotaxin hydrolyzes lysophosphatidylcholine to produce LPA where localized concentrations are able to signal via LPARs expressed by nearby cells, including cell types not producing the Autotaxin enzyme. Extracellular LPA production also appears tightly regulated with a half-life of approximately three minutes due to its rapid hydrolysis mediated by Lipid phosphate phosphatases (LPP) 1 and LPP3. The half-life of LPA increases 4 fold when intravenously introduced into mice deficient for LPP1 (lipid phosphate phosphohydrolase type 1), an enzyme that degrades LPA, and $L p p 1^{-1-}$ mice harbor higher levels of LPA (29).

Similar to S1P, LPA also signals via cognate GPCR receptors of which 6 LPA receptors $\left(\mathrm{LPA}_{1-6}\right)$ have been characterized and that are variably expressed on all immune populations (Figure 1). Thus, given its ability to signal extracellularly and act as an intracellular second messenger $(31,32)$, it is not surprising that LPA has been associated with a number of physiological processes including smooth muscle contraction, platelet aggregation, and blood pressure (33-35). Systemic changes in LPA levels have been observed in pregnancy (36), aging (37), and between sexes where females have been reported to harbor significantly elevated LPA serum levels compared with males (37). Interestingly, all of these circumstances can be 


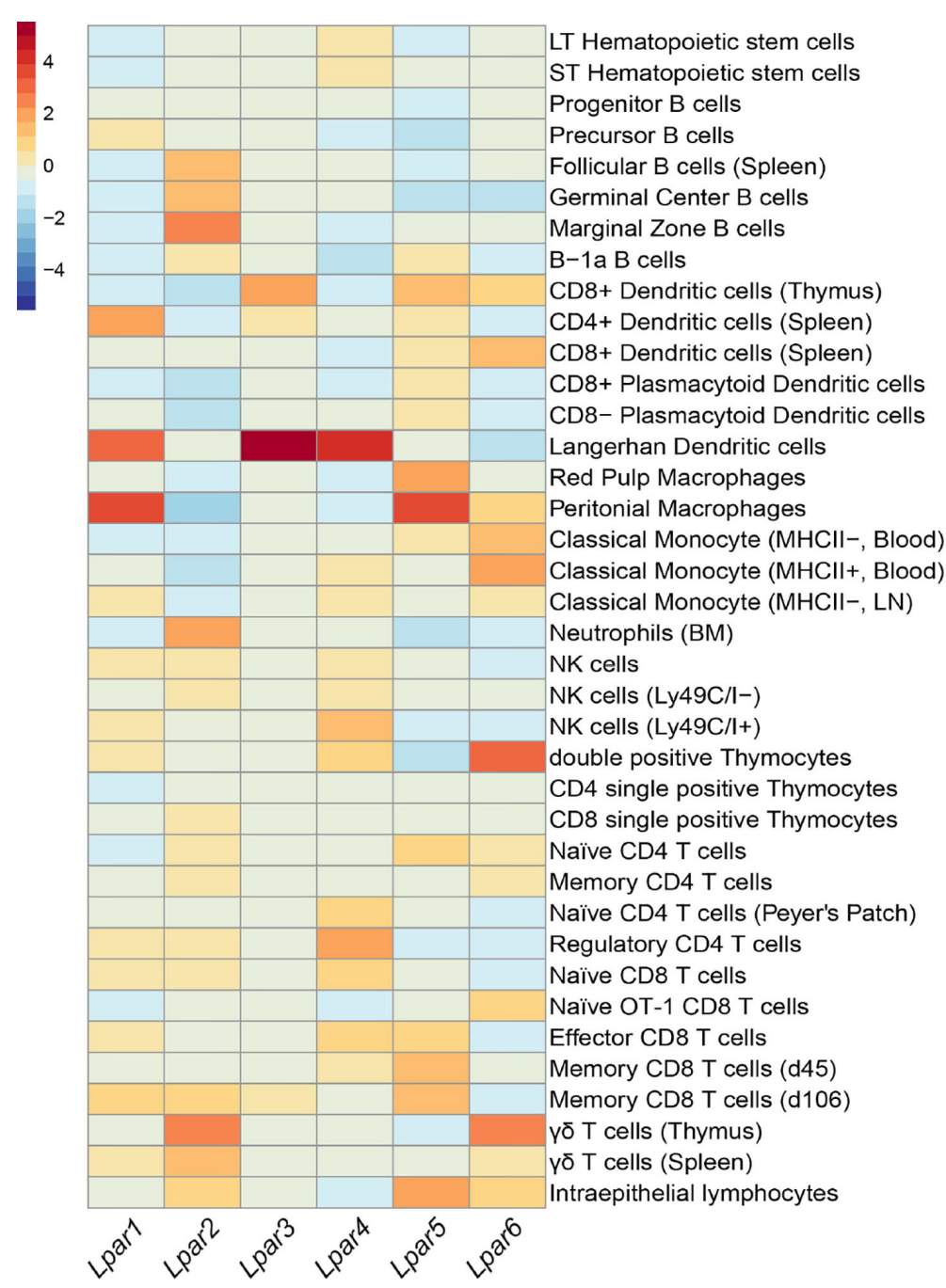

FIGURE 1 | Summary of Lpar expression in different leukocytes. Heatmap showing expression of LPA receptors across immune subsets. Data was compiled from Immune Genome Project microarray of sorted immune populations and scaled to columns (30). Color scale on the top left indicates level of mRNA expression. Unless specified, immune populations were sorted from 6-8 week C57BL/6 mice.

considered to require suppression of inflammation. However, its role in wound repair (38) would speak directly to the suppressive affect LPA signaling has on CD8 T cell function.

Resolution of a wound can be subdivided into 4 distinct phases: hemostasis, inflammation, proliferation, and remodeling (39) (Figure 2). Immediately after a physical trauma, platelets are first to arrive to help initiate the coagulation cascade and help activate fibroblasts and recruit both neutrophils and macrophages through the secretion of TGF $\beta$ (40). Interestingly, activated platelets are a potent source of LPA and soluble Autotaxin can associate with platelet integrins and produce LPA (41). Neutrophils are the initial immune cells to infiltrate wounds and help drive an inflammatory response to eliminate any microbes (42). Although short-lived cells, the infiltration of neutrophils is vital for the production of various growth factors like IL-17 and VEGF which help in the proliferation of fibroblasts, keratinocytes, and endothelial cells. Loss of early neutrophil recruitment delays epithelialization and decreases neovascularization at the site of injury (43). While LPA does not appear to have any direct effect on neutrophil migration per se, it is able to enhance the migratory response of neutrophils to suboptimal concentrations of $\mathrm{N}$ formyl-L-methionyl-L-leucyl-phenylalanine (fMLP) (46) suggesting a role for LPA in aiding neutrophil migration to sites of inflammation. Monocyte recruitment, and subsequent differentiation into macrophages, occurs 5 to $6 \mathrm{~h}$ post injury. Anti- inflammatory macrophages are involved with the secretion of TGF $\beta$, clearing cellular debris, helping reorganize the extracellular matrix (ECM) and contracting the wound. Macrophage-mediated degradation of the ECM leads to more endothelial proliferation and the release of angiogenesis factors such as FGF and placental growth factor (PIGF) (52). Of note, LPA is able to directly promote the conversion of monocytes to 


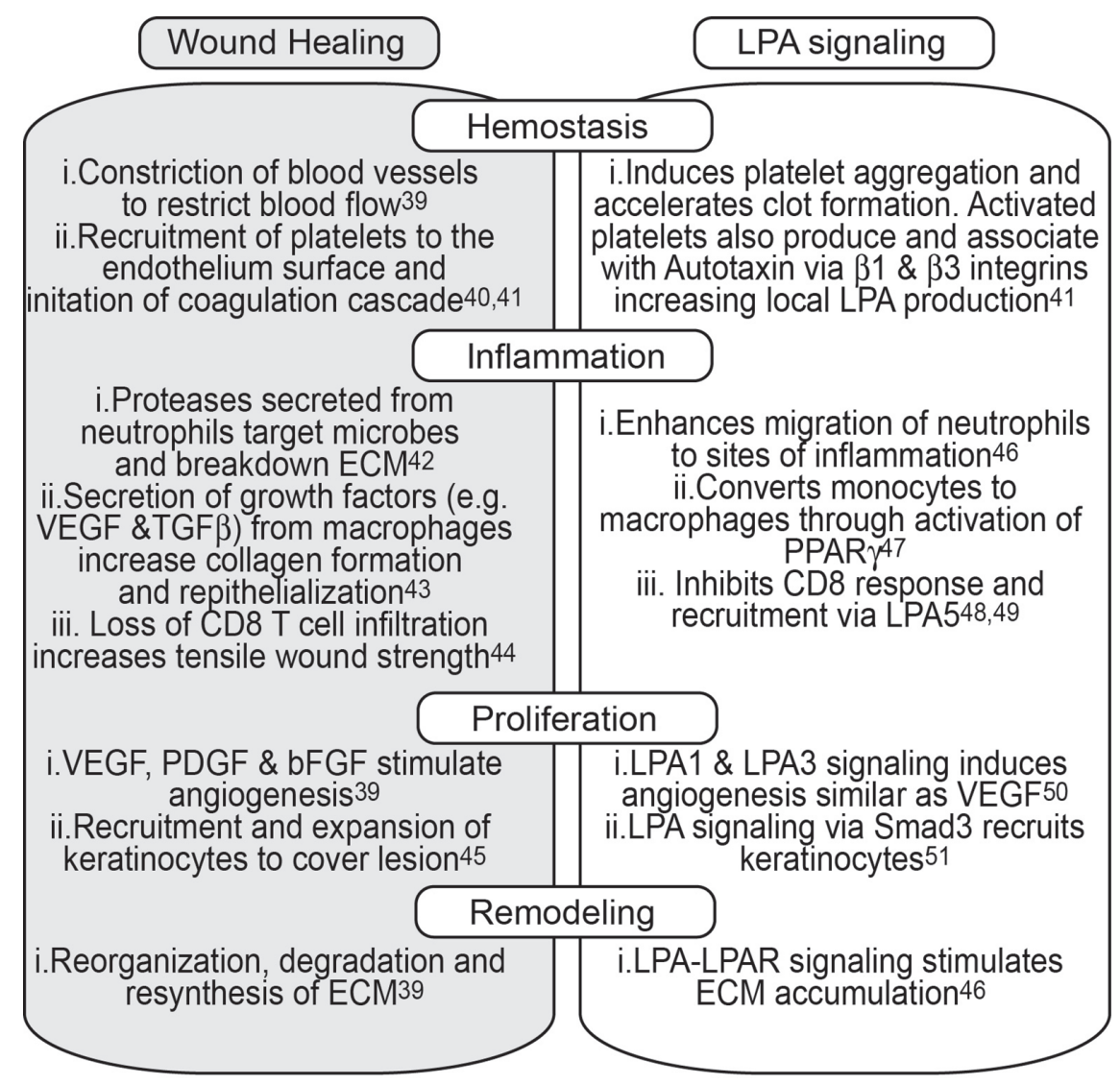

FIGURE 2 | The physiological role of LPA signaling in wound repair. A simplified version of the major biological processes that occur during each step of wound healing stratified across four major groups; Hemostasis, Inflammation, Proliferation, and Remodeling. Each group is further broken down to biological events that characterize each group (grey tile) and the role of LPA in each of those biological events (clear tile).

macrophages and is true in both humans and mice (47). In fact, culturing monocytes in media containing only LPA converts

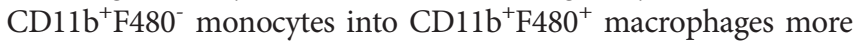
so than only M-CSF (47). While the role of T cells in wound repair remains largely unexplored, depletion of CD8 T cells increases tensile strength across lesions suggesting some inhibitory role of CD8 T cells (44). Given this overlap of LPA-mediated effects and wound healing, it is not surprising that topical application of LPA to physical wounds in rats or mice promoted accelerated healing with increased neoepithelial thickness $(53,54)$, an effect further seen in aged rats compared to young rats (55). As the recruitment and proliferation of keratinocytes remains critical for skin repair, LPA signaling not only induced increased migration and expansion of keratinocytes but also induced a four to eight fold increase in TGF $\alpha$ production (45).

\section{LPA AS A CANCER INTRINSIC GROWTH FACTOR}

Tumors have been appreciated to rely on pathways used in wound repair and have often been described as wounds that never heal
(56). For example, IL-1 $\beta$, IL-6, and IL- 8 are cytokines that are secreted during the early inflammatory response after tissue damage and are involved with re-epithelization. However, in breast cancer, these cytokines are associated with a poor prognosis as they have been linked to tumor growth and metastasis (57-59). Similarly, LPA has been exploited by cancers to promote growth in several non-redundant ways. LPA signaling has directly been linked to hTERT upregulation as ovarian cancer lines treated with LPA have increased telomerase activity as early as $12 \mathrm{~h}$ after co-culture (60). This replicative advantage mediated by LPA helps explain the increased expression of the gene encoding Autotaxin, ENPP2, observed in ovarian cancer stem cells; a population of long lasting malignant cells that seeds cancer growth (61). In fact, autocrine LPA signaling in these cells has been shown to promote sphere forming ability and upregulation of ALDH, markers associated with cancer stem cells. This dependence on LPA signaling by ovarian cancer cells provided rationale to explore the use of LPA as a potential biomarker in ovarian cancer progression and accounts for the high levels of LPA found in ascites fluid from individuals with ovarian cancer (62). LPA signaling can also directly affect several 'hallmarks' of cancer (63), including proliferation (64), or metastasis of colorectal 
cancers (65), increased angiogenesis in transformed NIH3T3 cells (66) and further demonstrated in chorio-allantoic membrane assays (50), resisting cell death by increasing insensitivity to chemotherapy in ovarian cancers (67), altered lipogenesis as LPA increases fatty acid synthase in ovarian cancers (68), and by modulating inflammation through activation of PPAR- $\gamma$ in the tumor stroma (69).

\section{LPAR SIGNALING AS A 'CHECKPOINT' IN THE ANTI-TUMOR RESPONSE}

Given the mechanisms by which CTLA- 4 and PD- 1 suppress T cell function, the LPA signaling axis can be considered as an additional form of suppression. However unlike CTLA-4, which inhibits activating signals of antigen presenting cells to CD8 $\mathrm{T}$ cells, or PD-1, which inhibits interactions of effector T cells and targets cells, we find LPA signaling disrupts T cell engagement with APCs and target cells $(48,49)$. In initial work from our lab, we demonstrated that LPA engagement with the LPA5 receptor induces a signal that inhibits TCR-induced $\mathrm{Ca}^{2+}$ release from intracellular stores in naïve CD8 T cells (48). This suppressive LPA signaling is dependent on the LPA5 receptor, as intracellular $\mathrm{Ca}^{2+}$ levels are not depressed in LPA5-deficient CD8 T cells after TCR signaling is induced in the presence of LPA $(48,49)$. Evidence in B lymphocytes strongly suggests LPA inhibition of antigen receptor signaling manifests via impaired $\mathrm{IP}_{3}$ receptor activity, thereby limiting the amount of $\mathrm{Ca}^{2+}$ released from ER stores after antigen receptor stimulation (70). Of note, the activity of antigen receptor proximal signaling molecules, e.g., tyrosine kinases and PLC $\gamma$, are unchanged in the presence of LPA (70). Thus, the mechanism of inhibition imposed by LPA5 on CD8 $\mathrm{T}$ cells differs from how PD- 1 and CTLA-4 suppress CD8 T cells. We have documented that LPA5 signals via $\mathrm{G \alpha}_{12 / 13^{-}}$ assoicated heterotrimeric G-proteins in lymphocytes and subsequently relies on the ARHGEF1 intracellular signaling effector molecule for antigen receptor mediated suppression of LPA (70). This is in contrast to CTLA-4 which can inhibit T cell function in the absence of intracellular signaling and PD-1 which depends on the recruitment of SHP-1 or SHP-2 for its suppressive action. Given the pleiotropic downstream effects of $\mathrm{Ca}^{2+}$ dependent processes that result from TCR signaling, it is perhaps not surprising that TCR-stimulated CD8 T cells fail to appropriately activate and proliferate in the presence of LPA both in vitro and in vivo (48). Moreover, in vivo LPA-induced suppression was not observed with CD8 T cells harboring null Lpar5 alleles (48). Importantly, we have more recently determined that in addition to suppressing TCR-induced cytosolic calcium mobilization, LPA also inhibits TCR-driven ERK activation (49) and both calcium and ERK have been previously shown to be required for granule exocytosis (71-74). Accordingly, in the presence of an LPA5 agonist, effector CD8 $\mathrm{T}$ cells display impaired perforin localization to the immunological synapse upon cognate antigen stimulation (49). These data demonstrate LPA engagement of LPA5 is able to suppress cells at different stages of CD8 T cell maturation and characteristic of other checkpoint regulators. As a consequence, Lpar5 $^{-/}$tumor-specific CD8 T cells are able to provide better control of tumor burden 8 days after adoptive transfer compared with wild type tumor-specific CD8 T cells $(48,49)$.

Given the negative regulation of CD8 T cells by an LPA-LPA5 axis, one might expect that a deficiency in LPA5 receptor expression or a reduction in systemic levels of LPA might lead to autoimmunity, as observed with CTLA-4-deficient mice (7-9). Interestingly, neither the $\operatorname{Lpar5}^{-/-}$nor Enpp2 $2^{+/-}$(ATX heterozygous) mice, which harbor half the normal levels of systemic LPA, appear to present with any obvious systemic inflammatory conditions, raising questions about the role of LPA as a suppressive lipid. However, we note that PD-1-deficient mice develop significantly less severe immune pathologies compared to $\mathrm{Ctla}^{-/-}$mice and neither the $\mathrm{Tigit}^{-/-}$nor CD $96^{-1}$ mice present with spontaneous disease (75).

Together, these findings highlight the different functions displayed by inhibitory receptors. Furthermore, as postulated by the 'tide model' (76), the existence of multiple costimulatory and coinhibitory receptors on $\mathrm{T}$ cells suggest that $\mathrm{T}$ cell signaling is finely tuned and responds to the microenvironmental context in which TCR signaling occurs. Thus, while certain signals appear more paramount (e.g., CD28, CTLA-4, PD-1), certain contexts reveal the dominance of some (inhibitory) receptor signaling over other signals, as evident by the greater expansion of autologous CD8 T cells with DCs with anti-LAG3 blockade over anti-PD-1 blockade (77). Given that a majority of monotherapy checkpoint blockade fails to induce tumor remission, we propose that inhibition of the LPA signaling axis represents another potential 'checkpoint' to target in combinational therapy.

As all immune cells express at least one LPA receptor, it is reasonable to consider that this bioactive lipid has a role in modulating antitumor function in other tumor infiltrating leukocytes. (Figure 3) (78-80, 83). In fact, the suppression mediated by LPA signaling can extend beyond CD8 $\mathrm{T}$ cells to other cells in the adaptive arm. LPA can impair the migration of CD4 T cells and even causes chemorepulsion in vitro in a LPA2dependent manner (82). Moreover, in the presence of LPA, stimulated human CD4 $\mathrm{T}$ cells can produce IL-13, a Th2 cytokine involved with the activation of myeloid derived suppressor cells; thus, the reduced CD4 T cells that do migrate to the tumor are still involved in maintaining a pro-tumor environment $(84,85)$. Unlike CD4 T cells, LPA can act as a chemoattractant to natural killer (NK) cells yet also impair effector function. LPA signaling though LPA2 can increase cAMP levels and activated protein kinase A which subsequently inhibits the release of perforin in NK cells (81). Inhibition of protein kinase A activation restores NK cell cytotoxicity in the presence of LPA, suggesting a mechanism by which tumor derived LPA can impair NK function. Similar to the of CD8 T cells, B cells signaling and function are also inhibited through LPA5 (70). Specifically, LPA signaling though an LPA5-G $\alpha_{12 / 13}-$ Arhgef1 axis results in reduced $\mathrm{Ca}^{2+}$ signaling, a mechanism similar to CD8 T cells. Functionally, LPA signaling reduced humoral responses to T1-2 antigens suggesting a conserved inhibitory signal in T and B cells mediated through LPA5 (70). Functional changes mediated through LPA signaling extends further than lymphocytes as dendritic cells, macrophages, and neutrophils are 


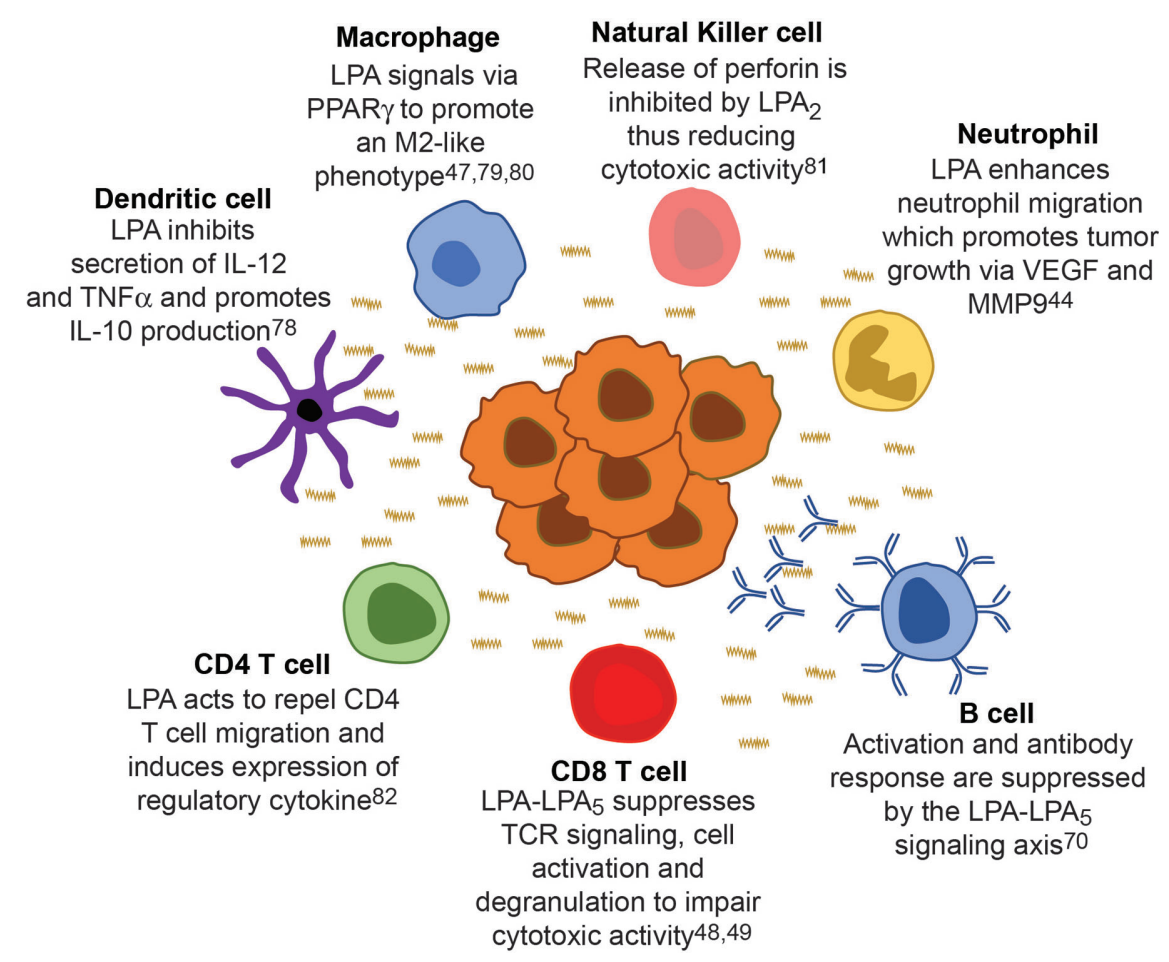

FIGURE 3 | Cancer derived LPA regulates immunity across all leukocytes. LPA (wavy line) originates from the tumor (center cellular mass) and suppresses the function of all leukocytes within the microenvironment creating a 'checkpoint' in the immune response against cancer. Each major leukocytes population is around the tumor mass with a specific example of how LPA signaling affects that immune cell, thereby generating a tumor promoting environment.

altered in the presence of this lipid. While LPA signaling does not affect endocytic function of dendritic cells, it does inhibit the secretion of IL-12 and TNF $\alpha$ while promoting the secretion of IL-10 (78). Thus, dendritic cells in the tumor microenvironment exposed to LPA would be poised for a tumor promoting function. Unlike CD4 T cells, LPA is a chemoattractant to neutrophils (46, 82). However this potenial influx of neutrophils has been linked to reduced overall survival in several cancers, presumably due to release of tumor promoting factors like VEGF or MMP9 (89). Futhermore, tumor infilitrating neutrophils can secrete TGF $\beta$ and have been implicated to changing the plasticity of macrophages to an M2-like state which promotes tumor growth (89). As LPA signaling though PPAR- $\gamma$ can cause the differentiation of monocytes to macrophages, we speculate the LPA signaling is involved with the influx and differentiation of tumor promoting myeloid cells $(47,79,80)$.

We believe LPA signaling has evolved to help with would repair to prevent an overactive immune response. Consistent with

\section{REFERENCES}

1. Serhan CN. Treating inflammation and infection in the 21st century: new hints from decoding resolution mediators and mechanisms. FASEB J (2017) 31:1273-88. doi: 10.1096/fj.201601222R

2. Zelenay S, van der Veen AG, Bottcher JP, Snelgrove KJ, Rogers N, Acton SE, et al. Cyclooxygenase-Dependent Tumor Growth through Evasion of Immunity. Cell (2015) 162:1257-70. doi: 10.1016/j.cell.2015.08.015 this, cancers exploit this very system to suppress immune cell function thereby representing a "checkpoint" in our endogenous antitumor response. We propose that manipulation of the LPA/ LPAR axis should be considered for potentially synergizing with anti-PD1, anti-CTLA4 or other therapies to improve leukocyte function in the tumor microenvironment (51).

\section{AUTHOR CONTRIBUTIONS}

DM and RT wrote and edited the review. All authors contributed to the article and approved the submitted version.

\section{FUNDING}

This work was funded by NIH/NIAID R01AI143261(RT), T32AI007405 (DM) and the Cancer League of Colorado (RT).

3. Walunas TL, Lenschow DJ, Bakker CY, Linsley PS, Freeman GJ, Green JM, et al. CTLA-4 can function as a negative regulator of $\mathrm{T}$ cell activation. Immunity (1994) 1:405-13. doi: 10.1016/1074-7613(94)90071-X

4. Qureshi OS, Zheng Y, Nakamura K, Attridge K, Manzotti C, Schmidt EM, et al. Trans-endocytosis of CD80 and CD86: a molecular basis for the cell-extrinsic function of CTLA-4. Science (2011) 332:600-3. doi: 10.1126/science.1202947

5. Ovcinnikovs V, Ross EM, Petersone L, Edner NM, Heuts F, Ntavli E, et al. CTLA-4-mediated transendocytosis of costimulatory molecules primarily 
targets migratory dendritic cells. Sci Immunol (2019) 4:eaaw0902 doi: 10.1126/sciimmunol.aaw0902

6. Tai X, Van Laethem F, Pobezinsky L, Guinter T, Sharrow SO, Adams A, et al. Basis of CTLA-4 function in regulatory and conventional CD4(+) T cells. Blood (2012) 119:5155-63. doi: 10.1182/blood-2011-11-388918

7. Wakamatsu E, Mathis D, Benoist C. Convergent and divergent effects of costimulatory molecules in conventional and regulatory CD4+ T cells. Proc Natl Acad Sci U S A (2013) 110:1023-8. doi: 10.1073/pnas.1220688110

8. Kong KF, Fu G, Zhang Y, Yokosuka T, Casas J, Canonigo-Balancio AJ, et al. Protein kinase C-eta controls CTLA-4-mediated regulatory T cell function. Nat Immunol (2014) 15:465-72. doi: 10.1038/ni.2866

9. Tivol EA, Borriello F, Schweitzer AN, Lynch WP, Bluestone JA, Sharpe AH. Loss of CTLA-4 leads to massive lymphoproliferation and fatal multiorgan tissue destruction, revealing a critical negative regulatory role of CTLA-4. Immunity (1995) 3:541-7. doi: 10.1016/1074-7613(95)90125-6

10. Waterhouse P, Penninger JM, Timms E, Wakeham A, Shahinian A, Lee KP, et al. Lymphoproliferative disorders with early lethality in mice deficient in Ctla-4. Science (1995) 270:985-8. doi: 10.1126/science.270.5238.985

11. Khattri R, Auger JA, Griffin MD, Sharpe AH, Bluestone JA. Lymphoproliferative disorder in CTLA-4 knockout mice is characterized by CD28-regulated activation of Th2 responses. J Immunol (1999) 162:5784-91.

12. Leach DR, Krummel MF, Allison JP. Enhancement of antitumor immunity by CTLA-4 blockade. Science (1996) 271:1734-6. doi: 10.1126/ science.271.5256.1734

13. Fecher LA, Agarwala SS, Hodi FS, Weber JS. Ipilimumab and its toxicities: a multidisciplinary approach. Oncologist (2013) 18:733-43. doi: 10.1634/ theoncologist.2012-0483

14. Zhang Y, Du X, Liu M, Tang F, Zhang P, Ai C, et al. Hijacking antibodyinduced CTLA-4 lysosomal degradation for safer and more effective cancer immunotherapy. Cell Res (2019) 29:609-27. doi: 10.1038/s41422-019-0184-1

15. Selby MJ, Engelhardt JJ, Quigley M, Henning KA, Chen T, Srinivasan M, et al. Anti-CTLA-4 antibodies of IgG2a isotype enhance antitumor activity through reduction of intratumoral regulatory T cells. Cancer Immunol Res (2013) 1:32-42. doi: 10.1158/2326-6066.CIR-13-0013

16. Simpson TR, Li F, Montalvo-Ortiz W, Sepulveda MA, Bergerhoff K, Arce F, et al. Fc-dependent depletion of tumor-infiltrating regulatory $\mathrm{T}$ cells codefines the efficacy of anti-CTLA-4 therapy against melanoma. J Exp Med (2013) 210:1695-710. doi: 10.1084/jem.20130579

17. Sharma A, Subudhi SK, Blando J, Vence L, Wargo J, Allison JP, et al. AntiCTLA-4 Immunotherapy Does Not Deplete FOXP3(+) Regulatory T Cells (Tregs) in Human Cancers-Response. Clin Cancer Res (2019) 25:3469-70. doi: 10.1158/1078-0432.CCR-19-0402

18. Chemnitz JM, Parry RV, Nichols KE, June CH, Riley JL. SHP-1 and SHP-2 associate with immunoreceptor tyrosine-based switch motif of programmed death 1 upon primary human $\mathrm{T}$ cell stimulation, but only receptor ligation prevents T cell activation. J Immunol (2004) 173:945-54. doi: 10.4049/ jimmunol.173.2.945

19. Sheppard KA, Fitz LJ, Lee JM, Benander C, George JA, Wooters J, et al. PD-1 inhibits T-cell receptor induced phosphorylation of the ZAP70/CD3zeta signalosome and downstream signaling to PKCtheta. FEBS Lett (2004) 574:37-41. doi: 10.1016/j.febslet.2004.07.083

20. Hui E, Cheung J, Zhu J, Su X, Taylor MJ, Wallweber HA, et al. T cell costimulatory receptor $\mathrm{CD} 28$ is a primary target for PD-1-mediated inhibition. Science (2017) 355:1428-33. doi: 10.1126/science.aaf1292

21. Taube JM, Anders RA, Young GD, Xu H, Sharma R, McMiller TL, et al. Colocalization of inflammatory response with B7-h1 expression in human melanocytic lesions supports an adaptive resistance mechanism of immune escape. Sci Transl Med (2012) 4:127ra37. doi: 10.1126/scitranslmed. 3003689

22. Sanmamed MF, Chen L. A Paradigm Shift in Cancer Immunotherapy: From Enhancement to Normalization. Cell (2018) 175:313-26. doi: 10.1016/ j.cell.2018.09.035

23. Ahn E, Araki K, Hashimoto M, Li W, Riley JL, Cheung J, et al. Role of PD-1 during effector CD8 T cell differentiation. Proc Natl Acad Sci U S A (2018) 115:4749-54. doi: 10.1073/pnas.1718217115

24. Wei SC, Duffy CR, Allison JP. Fundamental Mechanisms of Immune Checkpoint Blockade Therapy. Cancer Discov (2018) 8:1069-86. doi: 10.1158/2159-8290.CD-18-0367
25. Cyster JG, Schwab SR. Sphingosine-1-phosphate and lymphocyte egress from lymphoid organs. Annu Rev Immunol (2012) 30:69-94. doi: 10.1146/annurevimmunol-020711-075011

26. Hashimoto T, Okudaira S, Igarashi K, Hama K, Yatomi Y, Aoki J. Identification and biochemical characterization of a novel autotaxin isoform, ATXdelta, with a four-amino acid deletion. J Biochem (2012) 151:89-97. doi: 10.1093/jb/mvr126

27. Hausmann J, Kamtekar S, Christodoulou E, Day JE, Wu T, Fulkerson Z, et al. Structural basis of substrate discrimination and integrin binding by autotaxin. Nat Struct Mol Biol (2011) 18:198-204. doi: 10.1038/nsmb.1980

28. Perrakis A, Moolenaar WH. Autotaxin: structure-function and signaling. J Lipid Res (2014) 55:1010-8. doi: 10.1194/jlr.R046391

29. Tomsig JL, Snyder AH, Berdyshev EV, Skobeleva A, Mataya C, Natarajan V, et al. Lipid phosphate phosphohydrolase type 1 (LPP1) degrades extracellular lysophosphatidic acid in vivo. Biochem J (2009) 419:611-8. doi: 10.1042/ BJ20081888

30. Heng TS, Painter MW, Immunological Genome Project C. The Immunological Genome Project: networks of gene expression in immune cells. Nat Immunol (2008) 9:1091-4. doi: 10.1038/ni1008-1091

31. Bektas M, Payne SG, Liu H, Goparaju S, Milstien S, Spiegel S. A novel acylglycerol kinase that produces lysophosphatidic acid modulates cross talk with EGFR in prostate cancer cells. J Cell Biol (2005) 169:801-11. doi: 10.1083/ jcb.200407123

32. Kalari S, Zhao Y, Spannhake EW, Berdyshev EV, Natarajan V. Role of acylglycerol kinase in LPA-induced IL-8 secretion and transactivation of epidermal growth factor-receptor in human bronchial epithelial cells. Am J Physiol Lung Cell Mol Physiol (2009) 296:L328-36. doi: 10.1152/ ajplung.90431.2008

33. Kano K, Matsumoto H, Inoue A, Yukiura H, Kanai M, Chun J, et al. Molecular mechanism of lysophosphatidic acid-induced hypertensive response. Sci Rep (2019) 9:2662. doi: 10.1038/s41598-019-39041-4

34. Leblanc R, Houssin A, Peyruchaud O. Platelets, autotaxin and lysophosphatidic acid signalling: win-win factors for cancer metastasis. $\mathrm{Br} J$ Pharmacol (2018) 175:3100-10. doi: 10.1111/bph.14362

35. Smyth SS, Cheng HY, Miriyala S, Panchatcharam M, Morris AJ. Roles of lysophosphatidic acid in cardiovascular physiology and disease. Biochim Biophys Acta (2008) 1781:563-70. doi: 10.1016/j.bbalip.2008.05.008

36. Tokumura A, Kanaya Y, Miyake M, Yamano S, Irahara M, Fukuzawa K. Increased production of bioactive lysophosphatidic acid by serum lysophospholipase D in human pregnancy. Biol Reprod (2002) 67:1386-92. doi: 10.1095/biolreprod.102.004051

37. Hosogaya S, Yatomi Y, Nakamura K, Ohkawa R, Okubo S, Yokota H, et al. Measurement of plasma lysophosphatidic acid concentration in healthy subjects: strong correlation with lysophospholipase D activity. Ann Clin Biochem (2008) 45:364-8. doi: 10.1258/acb.2008.007242

38. Benesch MG, Zhao YY, Curtis JM, McMullen TP, Brindley DN. Regulation of autotaxin expression and secretion by lysophosphatidate and sphingosine 1phosphate. J Lipid Res (2015) 56:1134-44. doi: 10.1194/jlr.M057661

39. Larouche J, Sheoran S, Maruyama K, Martino MM. Immune Regulation of Skin Wound Healing: Mechanisms and Novel Therapeutic Targets. Adv Wound Care (New Rochelle) (2018) 7:209-31. doi: 10.1089/ wound.2017.0761

40. Rossaint J, Margraf A, Zarbock A. Role of Platelets in Leukocyte Recruitment and Resolution of Inflammation. Front Immunol (2018) 9:2712. doi: 10.3389/ fimmu.2018.02712

41. Fulkerson Z, Wu T, Sunkara M, Kooi CV, Morris AJ, Smyth SS. Binding of autotaxin to integrins localizes lysophosphatidic acid production to platelets and mammalian cells. J Biol Chem (2011) 286:34654-63. doi: 10.1074/ jbc.M111.276725

42. Wilgus TA, Roy S, McDaniel JC. Neutrophils and Wound Repair: Positive Actions and Negative Reactions. Adv Wound Care (New Rochelle) (2013) 2:379-88. doi: 10.1089/wound.2012.0383

43. Su Y, Richmond A. Chemokine Regulation of Neutrophil Infiltration of Skin Wounds. Adv Wound Care (New Rochelle) (2015) 4:631-40. doi: 10.1089/ wound.2014.0559

44. Davis PA, Corless DJ, Aspinall R, Wastell C. Effect of CD4(+) and CD8(+) cell depletion on wound healing. Br J Surg (2001) 88:298-304. doi: 10.1046/j.13652168.2001.01665.x 
45. Piazza GA, Ritter JL, Baracka CA. Lysophosphatidic acid induction of transforming growth factors alpha and beta: modulation of proliferation and differentiation in cultured human keratinocytes and mouse skin. Exp Cell Res (1995) 216:51-64. doi: 10.1006/excr.1995.1007

46. Gerrard JM, Clawson CC, White JG. Lysophosphatidic acids: III. Enhancement of neutrophil chemotaxis. Am J Pathol (1980) 100:609-18.

47. Ray R, Rai V. Lysophosphatidic acid converts monocytes into macrophages in both mice and humans. Blood (2017) 129:1177-83. doi: 10.1182/blood-201610-743757

48. Oda SK, Strauch P, Fujiwara Y, Al-Shami A, Oravecz T, Tigyi G, et al. Lysophosphatidic acid inhibits CD8 T cell activation and control of tumor progression. Cancer Immunol Res (2013) 1:245-55. doi: 10.1158/23266066.CIR-13-0043-T

49. Mathew D, Kremer KN, Strauch P, Tigyi G, Pelanda R, Torres RM. LPA5 Is an Inhibitory Receptor That Suppresses CD8 T-Cell Cytotoxic Function via Disruption of Early TCR Signaling. Front Immunol (2019) 10:1159. doi: 10.3389/fimmu.2019.01159

50. Rivera-Lopez CM, Tucker AL, Lynch KR. Lysophosphatidic acid (LPA) and angiogenesis. Angiogenesis (2008) 11:301-10. doi: 10.1007/s10456-0089113-5

51. Sauer B, Vogler R, Zimmermann K, Fujii M, Anzano MB, Schafer-Korting M, et al. Lysophosphatidic acid interacts with transforming growth factor-beta signaling to mediate keratinocyte growth arrest and chemotaxis. J Invest Dermatol (2004) 123:840-9. doi: 10.1111/j.0022-202X.2004.23458.x

52. Kalucka J, Bierhansl L, Wielockx B, Carmeliet P, Eelen G. Interaction of endothelial cells with macrophages-linking molecular and metabolic signaling. Pflugers Arch (2017) 469:473-83. doi: 10.1007/s00424-0171946-6

53. Balazs L, Okolicany J, Ferrebee M, Tolley B, Tigyi G. Topical application of the phospholipid growth factor lysophosphatidic acid promotes wound healing in vivo. Am J Physiol Regul Integr Comp Physiol (2001) 280:R466-72. doi: 10.1152/ajpregu.2001.280.2.R466

54. Demoyer JS, Skalak TC, Durieux ME. Lysophosphatidic acid enhances healing of acute cutaneous wounds in the mouse. Wound Repair Regener (2000) 8:530-7. doi: 10.1046/j.1524-475x.2000.00530.x

55. Rhim JH, Jang IS, Kwon ST, Song KY, Yeo EJ, Park SC. Activation of wound healing in aged rats by altering the cellular mitogenic potential. J Gerontol A Biol Sci Med Sci (2010) 65:704-11. doi: 10.1093/gerona/glq065

56. Dvorak HF. Tumors: wounds that do not heal-redux. Cancer Immunol Res (2015) 3:1-11. doi: 10.1158/2326-6066.CIR-14-0209

57. Benoy IH, Salgado R, Van Dam P, Geboers K, Van Marck E, Scharpe S, et al. Increased serum interleukin- 8 in patients with early and metastatic breast cancer correlates with early dissemination and survival. Clin Cancer Res (2004) 10:7157-62. doi: 10.1158/1078-0432.CCR-04-0812

58. Cho YA, Sung MK, Yeon JY, Ro J, Kim J. Prognostic role of interleukin-6, interleukin-8, and leptin levels according to breast cancer subtype. Cancer Res Treat (2013) 45:210-9. doi: 10.4143/crt.2013.45.3.210

59. Dethlefsen C, Hojfeldt G, Hojman P. The role of intratumoral and systemic IL-6 in breast cancer. Breast Cancer Res Treat (2013) 138:657-64. doi: $10.1007 / \mathrm{s} 10549-013-2488-\mathrm{Z}$

60. Yang K, Zheng D, Deng X, Bai L, Xu Y, Cong YS. Lysophosphatidic acid activates telomerase in ovarian cancer cells through hypoxia-inducible factorlalpha and the PI3K pathway. J Cell Biochem (2008) 105:1194-201. doi: $10.1002 / j \mathrm{cb} .21919$

61. Seo EJ, Kwon YW, Jang IH, Kim DK, Lee SI, Choi EJ, et al. Autotaxin Regulates Maintenance of Ovarian Cancer Stem Cells through Lysophosphatidic Acid-Mediated Autocrine Mechanism. Stem Cells (2016) 34:551-64. doi: 10.1002/stem.2279

62. Cao L, Zhang Y, Fu Z, Dong L, Yang S, Meng W, et al. Diagnostic value of plasma lysophosphatidic acid levels in ovarian cancer patients: A case-control study and updated meta-analysis. J Obstet Gynaecol Res (2015) 41:1951-8. doi: 10.1111/jog.12806

63. Hanahan D, Weinberg RA. Hallmarks of cancer: the next generation. Cell (2011) 144:646-74. doi: 10.1016/j.cell.2011.02.013

64. Leve F, Peres-Moreira RJ, Binato R, Abdelhay E, Morgado-Diaz JA. LPA Induces Colon Cancer Cell Proliferation through a Cooperation between the ROCK and STAT-3 Pathways. PloS One (2015) 10:e139094. doi: 10.1371/ journal.pone.0139094
65. Shida D, Kitayama J, Yamaguchi H, Okaji Y, Tsuno NH, Watanabe T, et al. Lysophosphatidic acid (LPA) enhances the metastatic potential of human colon carcinoma DLD1 cells through LPA1. Cancer Res (2003) 63:1706-11.

66. Nam SW, Clair T, Kim YS, McMarlin A, Schiffmann E, Liotta LA, et al. Autotaxin (NPP-2), a metastasis-enhancing motogen, is an angiogenic factor. Cancer Res (2001) 61:6938-44.

67. Brindley DN, Lin FT, Tigyi GJ. Role of the autotaxin-lysophosphatidate axis in cancer resistance to chemotherapy and radiotherapy. Biochim Biophys Acta (2013) 1831:74-85. doi: 10.1016/j.bbalip.2012.08.015

68. Mukherjee A, Wu J, Barbour S, Fang X. Lysophosphatidic acid activates lipogenic pathways and de novo lipid synthesis in ovarian cancer cells. J Biol Chem (2012) 287:24990-5000. doi: 10.1074/jbc.M112.340083

69. Wahli W, Michalik L. PPARs at the crossroads of lipid signaling and inflammation. Trends Endocrinol Metab (2012) 23:351-63. doi: 10.1016/ j.tem.2012.05.001

70. Hu J, Oda SK, Shotts K, Donovan EE, Strauch P, Pujanauski LM, et al. Lysophosphatidic acid receptor 5 inhibits $\mathrm{B}$ cell antigen receptor signaling and antibody response. J Immunol (2014) 193:85-95. doi: 10.4049/jimmunol. 1300429

71. Lyubchenko TA, Wurth GA, Zweifach A. Role of calcium influx in cytotoxic T lymphocyte lytic granule exocytosis during target cell killing. Immunity (2001) 15:847-59. doi: 10.1016/S1074-7613(01)00233-3

72. Pores-Fernando AT, Zweifach A. Calcium influx and signaling in cytotoxic Tlymphocyte lytic granule exocytosis. Immunol Rev (2009) 231:160-73. doi: 10.1111/j.1600-065X.2009.00809.x

73. Robertson LK, Mireau LR, Ostergaard HL. A role for phosphatidylinositol 3kinase in TCR-stimulated ERK activation leading to paxillin phosphorylation and CTL degranulation. J Immunol (2005) 175:8138-45. doi: 10.4049/ jimmunol.175.12.8138

74. Takayama H, Sitkovsky MV. Antigen receptor-regulated exocytosis in cytotoxic T lymphocytes. J Exp Med (1987) 166:725-43. doi: 10.1084/ jem.166.3.725

75. Blake SJ, Dougall WC, Miles JJ, Teng MW, Smyth MJ. Molecular Pathways: Targeting CD96 and TIGIT for Cancer Immunotherapy. Clin Cancer Res (2016) 22:5183-8. doi: 10.1158/1078-0432.CCR-16-0933

76. Zhu Y, Yao S, Chen L. Cell surface signaling molecules in the control of immune responses: a tide model. Immunity (2011) 34:466-78. doi: 10.1016/ j.immuni.2011.04.008

77. Lichtenegger FS, Rothe M, Schnorfeil FM, Deiser K, Krupka C, Augsberger C, et al. Targeting LAG-3 and PD-1 to Enhance T Cell Activation by AntigenPresenting Cells. Front Immunol (2018) 9:385. doi: 10.3389/fimmu. 2018.00385

78. Panther E, Idzko M, Corinti S, Ferrari D, Herouy Y, Mockenhaupt M, et al. The influence of lysophosphatidic acid on the functions of human dendritic cells. J Immunol (2002) 169:4129-35. doi: 10.4049/jimmunol. 169.8.4129

79. Chawla A. Control of macrophage activation and function by PPARs. Circ Res (2010) 106:1559-69. doi: 10.1161/CIRCRESAHA.110.216523

80. Koh TJ, DiPietro LA. Inflammation and wound healing: the role of the macrophage. Expert Rev Mol Med (2011) 13:e23. doi: 10.1017/ S1462399411001943

83. Tazzyman S, Lewis CE, Murdoch C. Neutrophils: key mediators of tumour angiogenesis. Int $J$ Exp Pathol (2009) 90:222-31. doi: 10.1111/j.13652613.2009.00641.x

82. Knowlden SA, Capece T, Popovic M, Chapman TJ, Rezaee F, Kim M, et al. Regulation of T cell motility in vitro and in vivo by LPA and LPA2. PloS One (2014) 9:e101655. doi: 10.1371/journal.pone.0101655

84. Rubenfeld J, Guo J, Sookrung N, Chen R, Chaicumpa W, Casolaro V, et al. Lysophosphatidic acid enhances interleukin-13 gene expression and promoter activity in T cells. Am J Physiol Lung Cell Mol Physiol (2006) 290:L66-74. doi: 10.1152/ajplung.00473.2004

85. Suzuki A, Leland P, Joshi BH, Puri RK. Targeting of IL-4 and IL-13 receptors for cancer therapy. Cytokine (2015) 75:79-88. doi: 10.1016/j.cyto.2015. 05.026

81. Lagadari M, Truta-Feles K, Lehmann K, Berod L, Ziemer M, Idzko M, et al. Lysophosphatidic acid inhibits the cytotoxic activity of NK cells: involvement of Gs protein-mediated signaling. Int Immunol (2009) 21:667-77. doi: 10.1093/intimm/dxp035 
86. Jensen HK, Donskov F, Marcussen N, Nordsmark M, Lundbeck F, von der Maase H. Presence of intratumoral neutrophils is an independent prognostic factor in localized renal cell carcinoma. J Clin Oncol (2009) 27:4709-17. doi: 10.1200/JCO.2008.18.9498

87. Jensen TO, Schmidt H, Moller HJ, Donskov F, Hoyer M, Sjoegren P, et al. Intratumoral neutrophils and plasmacytoid dendritic cells indicate poor prognosis and are associated with pSTAT3 expression in AJCC stage I/II melanoma. Cancer (2012) 118:2476-85. doi: 10.1002/cncr.26511

88. Zou JM, Qin J, Li YC, Wang Y, Li D, Shu Y, et al. IL-35 induces N2 phenotype of neutrophils to promote tumor growth. Oncotarget (2017) 8:33501-14. doi: 10.18632 /oncotarget.16819

89. Zhang F, Wang H, Wang X, Jiang G, Liu H, Zhang G, et al. TGF-beta induces M2-like macrophage polarization via SNAIL-mediated suppression of a pro- inflammatory phenotype. Oncotarget (2016) 7:52294-306. doi: 10.18632/ oncotarget.10561

Conflict of Interest: The authors declare that the research was conducted in the absence of any commercial or financial relationships that could be construed as a potential conflict of interest.

Copyright (C) 2021 Mathew and Torres. This is an open-access article distributed under the terms of the Creative Commons Attribution License (CC BY). The use, distribution or reproduction in other forums is permitted, provided the original author(s) and the copyright owner(s) are credited and that the original publication in this journal is cited, in accordance with accepted academic practice. No use, distribution or reproduction is permitted which does not comply with these terms. 